\title{
Article \\ Closed-Form Solutions of Linear Ordinary Differential Equations with General Boundary Conditions
}

\author{
Efthimios Providas ${ }^{1, *}$, Stefanos Zaoutsos ${ }^{2}$ and Ioannis Faraslis ${ }^{1}$ (D) \\ 1 Department of Environmental Sciences, Gaiopolis Campus, University of Thessaly, 41500 Larissa, Greece; \\ faraslis@uth.gr \\ 2 Department of Energy Systems, Gaiopolis Campus, University of Thessaly, 41500 Larissa, Greece; \\ szaoutsos@uth.gr \\ * Correspondence: providas@uth.gr; Tel.: +30-2410-684-473
}

check for

updates

Citation: Providas, E.; Zaoutsos, S.; Faraslis, I. Closed-Form Solutions of Linear Ordinary Differential Equations with General Boundary Conditions. Axioms 2021, 10, 226. https: / / doi.org/10.3390/ axioms10030226

Academic Editors: Davron

Aslonqulovich Juraev

and Samad Noeiaghdam

Received: 31 July 2021

Accepted: 10 September 2021

Published: 14 September 2021

Publisher's Note: MDPI stays neutra with regard to jurisdictional claims in published maps and institutional affiliations.

Copyright: (c) 2021 by the authors. Licensee MDPI, Basel, Switzerland. This article is an open access article distributed under the terms and conditions of the Creative Commons Attribution (CC BY) license (https:// creativecommons.org/licenses/by/ $4.0 /)$.

\begin{abstract}
This paper deals with the solution of boundary value problems for ordinary differential equations with general boundary conditions. We obtain closed-form solutions in a symbolic form of problems with the general $n$-th order differential operator, as well as the composition of linear operators. The method is based on the theory of the extensions of linear operators in Banach spaces.
\end{abstract}

Keywords: differential equations; differential operators; non-local boundary value problems; general conditions; integral conditions; multipoint conditions; composition of operators

\section{Introduction}

Differential equations model numerous phenomena and processes in sciences and engineering. Boundary value problems for elementary differential equations with classical boundary conditions have been studied exhaustively by many researchers and comprehensive material is now included in various standard texts. A more difficult and less investigated subject is the general or nonlocal boundary value problems. In many applications, the incorporation of general boundary conditions such as multipoint and integral conditions is inevitable. For example, in [1], the necessity of integral conditions in certain models of epidemics and population growth and the effects when neglecting these conditions are explained.

Ordinary differential equations with non-local boundary conditions were first studied at the beginning of the 20th century in [2-4], and later in [5]. Abstract non-local boundary value problems were considered in [6-8]. Operator methods for solving differential equations are analyzed in the books [9-11]. A description of the theory and the different directions of differential equations with non-local boundary conditions are given in the monograph [12]. An overview of non-local boundary value problems and their historical evolution can also be found in the survey papers [13-16]. Boundary value problems with integral constraints have been considered in [17-25], to mention but a few. Boundary value problems with multipoint and integral conditions have been studied in [26-32], and others. The present paper aims at providing a framework for symbolic computations for the solution of linear ordinary differential equations of order $n$ with the most general multipoint and integral conditions, and boundary value problems for powers and products of differential operators.

In $C[a, b]$, let the general $n$-th order linear ordinary differential operator,

$$
A=a_{0}(x) \frac{d^{n}}{d x^{n}}+a_{1}(x) \frac{d^{n-1}}{d x^{n-1}}+\cdots+a_{n-1}(x) \frac{d}{d x}+a_{n}(x),
$$

where the coefficients $a_{i}(x), i=0, \ldots, n$, are all continuous functions on the interval $[a, b]$, $a_{0}(x) \neq 0$, and $D(A)=C^{n}[a, b]$ and $R(A)=C[a, b]$ are its domain and range, respectively. 
We are concerned with the solution in the closed-form of boundary value problem for the differential equation

$$
A u(x)=f(x), \quad x \in(a, b),
$$

and the boundary conditions

$$
Y(u)=\mathbf{b},
$$

where $\mathrm{Y}=\operatorname{col}\left(\mathrm{Y}_{1}, \ldots, \mathrm{Y}_{n}\right)$ is a vector of linear bounded functionals of the general form

$$
\mathrm{Y}_{i}(u)=\sum_{j=0}^{m} \sum_{k=0}^{n-1} v_{i k j} u^{(k)}\left(x_{j}\right)+\sum_{k=0}^{n-1} \int_{a}^{b} h_{i k}(t) u^{(k)}(t) d t, \quad i=1, \ldots, n,
$$

where $Y_{i} \in\left[C^{n-1}[a, b]\right]^{*}, i=1, \ldots, n$; the $m+1$ ordered points $a \leq x_{0}<x_{1}<\cdots<x_{m} \leq b$ are fixed boundary points, $h_{i k}(x), i=1, \ldots, n, k=0, \ldots, n-1$, are continuous functions on $[a, b], v_{i k j}, i=1, \ldots, n, k=0, \ldots, n-1, j=0, \ldots, m$, are constants, and $u^{(k)}$ designates the $k$-th order derivative of $u$. The non-homogeneous term $f(x) \in C[a, b]$, while the nonhomogeneous term $\mathbf{b}=\operatorname{col}\left(\beta_{1}, \ldots, \beta_{n}\right)$ is a constant vector. The function $u(x) \in C^{n}[a, b]$ is the sought solution. We formulate the above problem in a convenient symbolic form and establish uniqueness solvability criteria and derive the solution in closed-form. Solution formulae to some special boundary value problems for composite differential operators are also obtained. The method is based on the theory of the extensions of linear operators in Banach spaces, see, for example, [33-36], and is an extension of the work [37] by the authors.

The paper is organized as follows. In Section 2, we give some results needed for the analysis in later sections. Sections 3 and 4 contain the main findings of our investigation. In Section 5, the implementation of the technique is explained by solving two example problems. Finally, some conclusions are quoted in Section 6.

\section{Preliminaries}

Let $X, Y$ be Banach spaces and $P: X \rightarrow Y$ a linear operator. The operator $P$ is injective or one-to-one if for every $u_{1}, u_{2} \in D(P), u_{1} \neq u_{2}$ implies $P u_{1} \neq P u_{2}$. The operator $P$ is surjective or onto $Y$ if $R(P)=Y$. If $P$ is both injective and onto, then there exists the inverse operator $P^{-1}: Y \rightarrow X$ defined by $P^{-1} f=u$ if and only if $P u=f$ for each $f \in Y$; in this case, $R\left(P^{-1}\right)=D(P)$.

The operator $P$ is called closed if for every sequence $u_{m}$ in $D(P)$ converging to $u_{0}$ with $P u_{m} \rightarrow y_{0}, y_{0} \in Y$, it follows that $u_{0} \in D(P)$ and $P u_{0}=y_{0}$. A closed operator $P$ is called maximal if $R(P)=Y$ and $\operatorname{ker} P \neq\{0\}$.

The operator $P$ is correct if it is both injective and onto, and the inverse operator $P^{-1}$ is bounded on $Y$. The problem $P u=f$ is correct if the operator $P$ is correct.

An operator $P_{r}: X \rightarrow Y$ is a restriction of $P$, or $P$ is an extension of $P_{r}$, if $D\left(P_{r}\right) \subset D(P)$ and $P_{r} u=P u$ for all $u \in D\left(P_{r}\right)$.

Let $\Psi=\operatorname{col}\left(\Psi_{1}, \ldots, \Psi_{n}\right)$ be a column vector of functionals $\Psi_{i} \in X^{*}, i=1, \ldots, n$, and $\mathbf{v}=\left(v_{1}, \ldots, v_{n}\right)$ a row vector of elements $v_{i} \in X, i=1, \ldots, n$. By $\Psi(\mathbf{v})$, we symbolize the $n \times n$ matrix

$$
\Psi(\mathbf{v})=\left(\begin{array}{ccc}
\Psi_{1}\left(v_{1}\right) & \cdots & \Psi_{1}\left(v_{n}\right) \\
\vdots & \ddots & \vdots \\
\Psi_{n}\left(v_{1}\right) & \cdots & \Psi_{n}\left(v_{n}\right)
\end{array}\right)
$$

whose $\Psi_{i}\left(v_{j}\right)$ element is the value of the functional $\Psi_{i}$ on the element $v_{j}$. It is easy to show that

$$
\Psi(\mathbf{v N})=\Psi(\mathbf{v}) \mathbf{N} .
$$

where $\mathbf{N}$ is a $n \times m$ constant matrix. 
Proposition 1. Let $X, Y$ be real Banach spaces, $A: X \stackrel{\text { on }}{\rightarrow} Y$ a linear operator, $\mathbf{z}=\left(z_{1}, \ldots, z_{n}\right) a$ basis of $\operatorname{ker} A$, and $\widehat{A}$ the restriction of $A$ defined by

$$
\widehat{A} \subset A, \quad D(\widehat{A})=\{u: u \in D(A), \Phi(u)=\mathbf{0}\},
$$

where the components of the vector $\Phi=\operatorname{col}\left(\Phi_{1}, \ldots, \Phi_{n}\right)$ are linear bounded functionals on $X$. Then:

(i) The operator $\widehat{A}$ is injective if and only if

$$
\operatorname{det} \Phi(\mathbf{z})=\operatorname{det}\left(\begin{array}{ccc}
\Phi_{1}\left(z_{1}\right) & \ldots & \Phi_{1}\left(z_{n}\right) \\
\vdots & \ddots & \vdots \\
\Phi_{n}\left(z_{1}\right) & \ldots & \Phi_{n}\left(z_{n}\right)
\end{array}\right) \neq 0 .
$$

(ii) If additionally to (i), the operator $\widehat{A}^{-1}$ is bounded on the whole $Y$, then the operator $\widehat{A}$ is correct.

Proof. (i) Let $\operatorname{det} \Phi(\mathbf{z}) \neq 0$. Take $u_{0} \in \operatorname{ker} \widehat{A}$, then $\widehat{A} u_{0}=0, \Phi\left(u_{0}\right)=\mathbf{0}$ and

$$
u_{0}=\mathbf{z a}=a_{1} z_{1}+\cdots+a_{n} z_{n},
$$

where $\mathbf{a}=\operatorname{col}\left(a_{1}, \ldots, a_{n}\right) \in \mathbb{R}_{n}$. Acting by the vector $\Phi$ on $u_{0}$, we get

$$
\Phi\left(u_{0}\right)=\Phi(\mathbf{z a})=\Phi(\mathbf{z}) \mathbf{a}=\mathbf{0} .
$$

Since $\operatorname{det} \Phi(\mathbf{z}) \neq 0$ by hypothesis, it is implied that $\mathbf{a}=\mathbf{0}$ and so $u_{0}=0$. That is $\operatorname{ker} \widehat{A}=\{0\}$, and therefore $\widehat{A}$ is injective. Conversely, let $\operatorname{det} \Phi(\mathbf{z})=0$. Then, there exists a nonzero vector $\mathbf{c}=\operatorname{col}\left(c_{1}, \ldots, c_{n}\right) \in \mathbf{R}_{n}$ such that $\Phi(\mathbf{z}) \mathbf{c}=\mathbf{0}$. Consider the element $u_{0}=\mathbf{z c}$. Note that $u_{0} \neq 0$, since the components of $\mathbf{z}$ are linearly independent, and that $\Phi\left(u_{0}\right)=\Phi(\mathbf{z}) \mathbf{c}=\mathbf{0}$. This means that $u_{0} \in D(\widehat{A})$. Furthermore, $\widehat{A} u_{0}=A u_{0}=(A \mathbf{z}) \mathbf{c}=0$. From the above, it follows that $u_{0} \in \operatorname{ker} \widehat{A}$. Hence, $\widehat{A}$ is not injective.

(ii) Since $\widehat{A}$ is injective and the operator $\widehat{A}^{-1}$ is bounded on the whole $Y$ by hypothesis, it follows that the operator $\widehat{A}$ is correct.

Proposition 2. Let $X, Y$ be real Banach spaces, $A: X \stackrel{\text { on }}{\rightarrow} Y$ a linear operator, and $\mathbf{z}=\left(z_{1}, \ldots, z_{n}\right)$ a basis of ker $A$. If there exists a correct restriction $\widehat{A}$ of $A$ defined by

$$
\widehat{A} \subset A, \quad D(\widehat{A})=\{u: u \in D(A), \Phi(u)=\mathbf{0}\},
$$

where the components of the vector $\Phi=\operatorname{col}\left(\Phi_{1}, \ldots, \Phi_{n}\right)$ are linear bounded functionals on $X$, then $A$ is closed and so $A$ is a maximal operator.

Proof. Let $u_{m} \in D(A), u_{m} \rightarrow u$ and $A u_{m} \rightarrow y, m \rightarrow \infty$. Denote $A u_{m}=y_{m}$. Since $A$ is a linear operator and $\widehat{A}^{-1} y_{m}$ is a particular solution of $A u_{m}=y_{m}$, then every solution $u_{m}$ to this equation can be represented as

$$
u_{m}=\widehat{A}^{-1} y_{m}+\mathbf{z a}_{m}=\widehat{A}^{-1} y_{m}+a_{m 1} z_{1}+\cdots+a_{m n} z_{n},
$$

where $\mathbf{a}_{m}=\operatorname{col}\left(a_{m 1}, \ldots, a_{m n}\right) \in \mathbb{R}_{n}$. Acting by the vector $\Phi$ on (6), we obtain

$$
\Phi\left(u_{m}\right)=\Phi(\mathbf{z}) \mathbf{a}_{m} .
$$

Since, by hypothesis, the operator $\widehat{A}$ is correct, it follows from Proposition 1 that $\operatorname{det} \Phi(z) \neq 0$ and hence

$$
\mathbf{a}_{m}=\Phi^{-1}(\mathbf{z}) \Phi\left(u_{m}\right) .
$$


Substitution of (7) into (6) yields

$$
u_{m}=\widehat{A}^{-1} y_{m}+\mathbf{z} \Phi^{-1}(\mathbf{z}) \Phi\left(u_{m}\right) .
$$

Since $\widehat{A}^{-1}, \Phi_{1}, \ldots, \Phi_{n}$ are bounded and $u_{m} \rightarrow u, y_{m} \rightarrow y$ for $m \rightarrow \infty$, it follows that

$$
u=\widehat{A}^{-1} y+\mathbf{z} \Phi^{-1}(\mathbf{z}) \Phi(u) .
$$

Further, taking into account that $D(A)=D(\widehat{A}) \oplus \operatorname{ker} A$ [34], we conclude that $u \in D(A)$ and $A u=y$. So the operator $A$ is closed and hence $A$ is a maximal operator.

\section{General Boundary Conditions}

In this Section, we study boundary value problems for ordinary differential equations with general homogeneous and nonhomogeneous boundary conditions.

Let now $X=Y=C[a, b]$ and $X^{n}=C^{n}[a, b]$. Let $A: X \rightarrow X$ be the $n$-th order linear operator in (1), $\widehat{A}: X \rightarrow X$ a correct restriction of $A$ defined by

$$
\begin{aligned}
\widehat{A} u(x) & =A u(x), \quad x \in(a, b) \\
D(\widehat{A}) & =\{u: u \in D(A), \Phi(u)=\mathbf{0}\},
\end{aligned}
$$

where $\Phi=\operatorname{col}\left(\Phi_{1}, \ldots, \Phi_{n}\right)$ is a vector of $n$ linear bounded functionals $\Phi_{i} \in\left[X^{n-1}\right]^{*}$, $i=1, \ldots, n$, and $\widehat{A}^{-1}$ the inverse of $\widehat{A}$.

For example, the operator

$$
\begin{aligned}
\widehat{A} u(x) & =A u(x), \quad x \in(a, b) \\
D(\widehat{A}) & =\left\{u: u \in X^{n}, u\left(x_{0}\right)=u^{\prime}\left(x_{0}\right)=\cdots=u^{(n-1)}\left(x_{0}\right)=0\right\},
\end{aligned}
$$

where $\Phi_{i}(u)=u^{(i-1)}\left(x_{0}\right)=0, i=1, \ldots, n$, with $x_{0}$ being a fixed point in $[a, b]$, known as Cauchy boundary conditions, is correct. In the particular case where $A=\frac{d^{n}}{d x^{n}}$, the inverse $\widehat{A}$ and the unique solution of the correct problem $\widehat{A} u=f$, for any $f \in X$, is given explicitly by

$$
u=\widehat{A}^{-1} f=\frac{1}{(n-1) !} \int_{x_{0}}^{x}(x-t)^{n-1} f(t) d t, \quad \forall f \in X .
$$

From the above and Proposition 2, it is concluded that the $n$-th order linear operator $A$ in (1) is closed and maximal.

\subsection{Homogeneous Boundary Conditions}

First, we consider the boundary value problem with homogeneous boundary conditions, namely

$$
\begin{aligned}
A_{0} u & =A u=f, \quad x \in(a, b), \\
D\left(A_{0}\right) & =\{u: u \in D(A), \mathrm{Y}(u)=\mathbf{0}\},
\end{aligned}
$$

where the linear operator $A_{0}: X \rightarrow X$ is a restriction of the $n$-th order linear operator $A$ in (1), the components of the vector $Y=\operatorname{col}\left(Y_{1}, \ldots, Y_{n}\right) \in\left[X_{n}^{n-1}\right]^{*}$ are as in (3), (4), and $f \in X$.

Lemma 1. The linear operator $A_{0}$ is a closed operator.

Proof. Let $u_{r}, r=1,2, \ldots$, be a sequence in $D\left(A_{0}\right), u_{r} \rightarrow u_{0}$ and $A_{0} u_{r} \rightarrow f$. Then, $A u_{r} \rightarrow f$ and since $A$ is a closed operator, we get that $u_{0} \in D(A)$ and $A u_{0}=f$. Moreover, since $\mathrm{Y}_{1}, \ldots, \mathrm{Y}_{n}$ are bounded functionals on $X$, we get

$$
0=\mathrm{Y}_{i}\left(u_{r}\right) \rightarrow \mathrm{Y}_{i}\left(u_{0}\right)=0, \quad i=1, \ldots, n, \quad r \rightarrow \infty .
$$


This is that $u_{0} \in D\left(A_{0}\right)$ and so $A u_{0}=A_{0} u_{0}=f$. Hence, $A_{0}$ is a closed operator.

Theorem 1. Let $A_{0}$ be the linear operator defined by $(9), \mathbf{z}=\left(z_{1}, \ldots, z_{n}\right)$ a basis of $\operatorname{ker} A$, and $\widehat{A}^{-1}$ the inverse of the correct operator $\widehat{A}$ in (8). Then:

(i) The operator $A_{0}$ is injective if and only if $\operatorname{det} Y(\mathbf{z}) \neq 0$.

(ii) In addition, under (i), the operator $A_{0}$ is correct and the unique solution to the boundary value problem (9), for every $f \in X$, is given by

$$
u=A_{0}^{-1} f=\widehat{A}^{-1} f-\mathbf{z} Y^{-1}(\mathbf{z}) \mathrm{Y}\left(\widehat{A}^{-1} f\right) .
$$

Proof. (i) Suppose $\operatorname{det} Y(\mathbf{z}) \neq 0$. Let $u \in \operatorname{ker} A_{0}$. Then, $A_{0} u=A u=0$ and $u=\mathbf{z c}$, where $\mathbf{c}=\operatorname{col}\left(c_{1}, \ldots, c_{n}\right)$ is a vector of arbitrary constants. Additionally, $\mathrm{Y}(u)=\mathrm{Y}(\mathbf{z c})=$ $\mathrm{Y}(\mathbf{z}) \mathbf{c}=\mathbf{0}$, which implies that $\mathbf{c}=\mathbf{0}$. That is $u=0$ and consequently ker $A_{0}=\{0\}$. This proves that $A_{0}$ is injective. Conversely, let $\operatorname{det} Y(\mathbf{z})=0$. Then, there exists a nonzero vector of constants $\mathbf{c}=\operatorname{col}\left(c_{1}, \ldots, c_{n}\right)$ such that $Y(\mathbf{z}) \mathbf{c}=\mathbf{0}$. Let the element $u_{0}=\mathbf{z c}$ and notice that $u_{0} \neq 0$ since the components of $\mathbf{z}$ are linearly independent. Then, $A_{0} u_{0}=A u_{0}=$ $A(\mathbf{z c})=(A \mathbf{z}) \mathbf{c}=0$ and $\mathrm{Y}\left(u_{0}\right)=\mathrm{Y}(\mathbf{z c})=\mathrm{Y}(\mathbf{z}) \mathbf{c}=\mathbf{0}$. That is $u_{0}=\mathbf{z c} \in \operatorname{ker} A_{0}$ and as a consequence $A_{0}$ is not injective.

(ii) Let $\operatorname{det} Y(\mathbf{z}) \neq 0$. Then, from statement (i) follows that the opeartor $A_{0}$ is injective and hence there exists the inverse operator $A_{0}^{-1}: R\left(A_{0}\right) \subseteq X \rightarrow X$. Since by Lemma 1 the operator $A_{0}$ is closed, it is implied that $A_{0}^{-1}$ is a closed operator. Furthermore, $\widehat{A}$ is a correct restriction of the linear operator $A$ and therefore the general solution of the problem $A u=f$, for every $f \in X$, may be written as follows

$$
u=\widehat{A}^{-1} f+\mathbf{z c},
$$

where $\mathbf{c}=\operatorname{col}\left(c_{1}, \ldots, c_{n}\right)$ is a vector of arbitrary constants. By requiring $u$ to satisfy the boundary conditions in (9), we have

$$
\mathrm{Y}(u)=\mathrm{Y}\left(\widehat{A}^{-1} f\right)+\mathrm{Y}(\mathbf{z}) \mathbf{c}=0, \quad \text { and hence } \mathbf{c}=-\mathrm{Y}^{-1}(\mathbf{z}) \mathrm{Y}\left(\widehat{A}^{-1} f\right) .
$$

Substitution of $\mathbf{c}$ into (11) yields (10), which is the unique solution of the boundary value problem $A_{0} u=f$. In addition, it follows that $R\left(A_{0}\right)=X$ and since $A_{0}^{-1}$ is a closed operator with $D\left(A_{0}^{-1}\right)=R\left(A_{0}\right)=X$, it is implied that $A_{0}^{-1}$ is bounded on $X$. This proves that the operator $A_{0}$ is correct.

\subsection{Non-Homogeneous Boundary Conditions}

Next, we consider the complete non-homogeneous boundary value problem

$$
\begin{aligned}
A_{1} u & =A u=f, \quad x \in(a, b) \\
D\left(A_{1}\right) & =\{u: u \in D(A), Y(u)=\mathbf{b}\},
\end{aligned}
$$

where $A_{1}: X \rightarrow X$ is a restriction of the $n$-th order linear operator $A$ in (1), the components of the vector $\mathrm{Y}=\operatorname{col}\left(\mathrm{Y}_{1}, \ldots, \mathrm{Y}_{n}\right) \in\left[\mathrm{X}_{n}^{n-1}\right]^{*}$ are as in (3), (4), $\mathbf{b}=\operatorname{col}\left(\beta_{1}, \ldots, \beta_{n}\right) \in \mathbb{R}_{n}$, and $f \in X$. It is noted that the operator $A_{1}$ is not linear, since its domain is a nonlinear set.

We state and prove the next theorem for the existence and construction of the unique solution of the boundary value problem (12).

Theorem 2. Let $A_{1}$ be the operator defined by (12), $\mathbf{z}=\left(z_{1}, \ldots, z_{n}\right)$ a basis of $\operatorname{ker} A, \widehat{A}^{-1}$ the inverse of the correct operator $\widehat{A}$ in (8), and $A_{0}$ the operator defined in (9). Then, the operator $A_{1}$ is injective if and only if $A_{0}$ is injective. In this case, for every $f \in X$ and $\mathbf{b} \in \mathbb{R}_{n}$, the unique solution of (12) is given by

$$
u=A_{1}^{-1} f=\widehat{A}^{-1} f+\mathbf{z} Y^{-1}(\mathbf{z})\left[\mathbf{b}-\mathrm{Y}\left(\widehat{A}^{-1} f\right)\right] .
$$


Proof. Suppose $A_{0}$ is injective. Then, $\operatorname{ker} A_{0}=\{0\}$ and $\operatorname{det} Y(\mathbf{z}) \neq 0$ by Theorem 1 . Let $u_{1}, u_{2} \in D\left(A_{1}\right)$ and $A_{1} u_{1}=A_{1} u_{2}=f$. That is,

$$
A_{1} u_{i}=A u_{i}=f, \quad \mathrm{Y}\left(u_{i}\right)=\mathbf{b}, \quad i=1,2,
$$

from which we get

$$
A_{1} u_{i}=A u_{i}=A\left(u_{i}-\mathbf{z} Y^{-1}(\mathbf{z}) \mathbf{b}\right)=f, \quad Y\left(u_{i}-\mathbf{z} Y^{-1}(\mathbf{z}) \mathbf{b}\right)=\mathbf{0}, \quad i=1,2,
$$

by taking into account that $A \mathbf{z}=\mathbf{0}$ and (5). From (14), it is implied that $u_{i}-\mathbf{z} Y^{-1}(\mathbf{z}) \mathbf{b} \in$ $D\left(A_{0}\right)$ and

$$
A_{1} u_{i}=A_{0}\left(u_{i}-\mathbf{z} Y^{-1}(\mathbf{z}) \mathbf{b}\right)=f, \quad i=1,2 .
$$

From Theorem 1, we have

$$
u_{i}-\mathbf{z} Y^{-1}(\mathbf{z}) \mathbf{b}=A_{0}^{-1} f \quad \text { or } \quad u_{i}=A_{0}^{-1} f+\mathbf{z} Y^{-1}(\mathbf{z}) \mathbf{b}, \quad i=1,2 .
$$

Since $A_{0}$ is injective, it is concluded that $u_{1}=u_{2}$ and therefore $A_{1}$ is an injective operator. Conversely, suppose $A_{1}$ is injective. Let $u \in \operatorname{ker} A_{1}$, which means

$$
A_{1} u=A u=0, \quad Y(u)=\mathbf{b}, \quad \mathbf{b} \in \mathbb{R}_{n} .
$$

It follows that $u=\mathbf{z c}$, where $\mathbf{c}$ is a vector of constants, and

$$
\mathrm{Y}(u)=\mathrm{Y}(\mathbf{z c})=\mathrm{Y}(\mathbf{z}) \mathbf{c}=\mathbf{b} .
$$

Since $A_{1}$ is injective, the system (16) has only one solution, that is $\operatorname{det} Y(\mathbf{z}) \neq 0$, and hence by Theorem $1 A_{0}$ is injective.

Finally, under the hypothesis that $A_{1}$ is injective, for any $u \in D\left(A_{1}\right)$ that solves the completely nonhomogeneous problem $A_{1} u=f$, we have

$$
A_{1} u=A u=A\left(u-\mathbf{z} Y^{-1}(\mathbf{z}) \mathbf{b}\right)=f, \quad Y\left(u-\mathbf{z Y}^{-1}(\mathbf{z}) \mathbf{b}\right)=\mathbf{0} .
$$

This means that $u-\mathbf{z} Y^{-1}(\mathbf{z}) \mathbf{b} \in D\left(A_{0}\right)$ and

$$
A_{1} u=A_{0}\left(u-\mathbf{z Y}^{-1}(\mathbf{z}) \mathbf{b}\right)=f .
$$

The solution to this problem follows from Theorem 1, namely

$$
\begin{aligned}
u-\mathbf{z} Y^{-1}(\mathbf{z}) \mathbf{b} & =A_{0}^{-1} f \\
& =\widehat{A}^{-1} f-\mathbf{z} Y^{-1}(\mathbf{z}) Y\left(\widehat{A}^{-1} f\right),
\end{aligned}
$$

from where we get

$$
\begin{aligned}
u & =\widehat{A}^{-1} f-\mathbf{z} Y^{-1}(\mathbf{z}) Y\left(\widehat{A}^{-1} f\right)+\mathbf{z} Y^{-1}(\mathbf{z}) \mathbf{b} \\
& =\widehat{A}^{-1} f+\mathbf{z} Y^{-1}(\mathbf{z})\left[\mathbf{b}-\mathrm{Y}\left(\widehat{A}^{-1} f\right)\right]
\end{aligned}
$$

\section{Composition of Operators}

In this Section, we investigate boundary value problems for special differential operators, specifically the $k$-th power of an operator and the product of two operators, with general homogeneous boundary conditions. 


\section{1. $k$-th Power of an Operator}

The $k$-th power of an operator $A^{k}$ is defined as the composition of the operator with itself, repeated $k$ times, i.e.,

$$
A^{k}=A\left(A^{k-1}\right)=\underbrace{A A \cdots A}_{k}, \quad k=2,3 \ldots
$$

If $A: X \rightarrow X$ is an $n$-th order linear differential operator with $D(A)=X^{n}$ then $A^{k}: X \rightarrow X$ is a $k n$-th order linear operator with $D\left(A^{k}\right)=X^{k n}$.

Let the boundary value problem

$$
\begin{aligned}
A_{0}^{k} u & =A^{k} u=f, \quad x \in(a, b), \\
D\left(A_{0}^{k}\right) & =\left\{u: u \in D\left(A^{k}\right), \mathrm{Y}(u)=\mathbf{0}, \mathrm{Y}(A u)=\mathbf{0}, \ldots, \mathrm{Y}\left(A^{k-1} u\right)=\mathbf{0}\right\},
\end{aligned}
$$

where the operator $A_{0}^{k}: X \rightarrow X$, the operators $A$ and $A_{0}$ are defined as in (1) and (9), respectively, the components of the vector $\mathrm{Y}=\operatorname{col}\left(\mathrm{Y}_{1}, \ldots, \mathrm{Y}_{n}\right)$ are as in (3), (4) where now $Y_{i} \in\left[X^{k n-1}\right]^{*}, i=1, \ldots, n$, and $f \in X$. We state the following theorem.

Theorem 3. Let $A_{0}^{k}$ be the linear operator defined in (18) and $\mathbf{z}=\left(z_{1}, \ldots, z_{n}\right)$ be a basis of $\operatorname{ker} A$. Then:

(i) The operator $A_{0}^{k}$ is injective if and only if $\operatorname{det} \mathrm{Y}(\mathbf{z}) \neq 0$.

(ii) Moreover, under (i), the operator $A_{0}^{k}$ is correct and the unique solution to the boundary value problem (18), for any $f \in X$, is given by

$$
u=\left(A_{0}^{k}\right)^{-1} f=A_{0}^{-k} f .
$$

Proof. (i) Let $\operatorname{det} Y(\mathbf{z}) \neq 0$. Then, by Theorem 1, the operator $A_{0}$ is injective. Further, the operator $A_{0}^{k}$ is injective as a composition of injective operators. Conversely, let $A_{0}^{k}$ be injective. Then, $\operatorname{ker} A_{0}^{k}=\{0\}$, and from the well known relation, which holds for any linear operator $A_{0}$,

$$
\text { ker } A_{0} \subseteq \operatorname{ker} A_{0}^{2} \subseteq \ldots \subseteq \operatorname{ker} A_{0}^{k}, \quad k \in N,
$$

follows that ker $A_{0}=\{0\}$, i.e., $A_{0}$ is injective. Then, by Theorem 1 , we have $\operatorname{det} Y(\mathbf{z}) \neq 0$.

(ii) Let $\operatorname{det} Y(\mathbf{z}) \neq 0$. Then, by Theorem 1 , the operator $A_{0}$ is correct. Observe that the problem (18), for any $f \in X$, by setting $A u=v_{1}, A v_{1}=v_{2}, \ldots, A v_{k-2}=v_{k-1}, A v_{k-1}=f$ can be decomposed into the $k$ boundary value problems:

$$
\begin{array}{rcll}
A v_{k-1}=f, & \mathrm{Y}\left(v_{k-1}\right)=\mathbf{0} & \text { or } & A_{0} v_{k-1}=f, \\
A v_{k-2}=v_{k-1}, & \mathrm{Y}\left(v_{k-2}\right)=\mathbf{0} & \text { or } & A_{0} v_{k-2}=v_{k-1}, \\
\cdots & \\
A v_{1}=v_{2}, & \mathrm{Y}\left(v_{1}\right)=\mathbf{0} & \text { or } & A_{0} v_{1}=v_{2}, \\
A u=v_{1}, & \mathrm{Y}(u)=\mathbf{0} & \text { or } & A_{0} u=v_{1} .
\end{array}
$$

By applying Theorem 1 successively, we get

$$
\begin{aligned}
v_{k-1}= & A_{0}^{-1} f=\widehat{A}^{-1} f-\mathbf{z} Y^{-1}(\mathbf{z}) \mathrm{Y}\left(\widehat{A}^{-1} f\right), \\
v_{k-2}= & A_{0}^{-1} v_{k-1}=A_{0}^{-1}\left(A_{0}^{-1} f\right)=A_{0}^{-2} f, \\
& \cdots \\
v_{1}= & A_{0}^{-1} v_{2}=A_{0}^{-1}\left(A_{0}^{-(k-2)} f\right)=A_{0}^{-(k-1)} f, \\
u= & A_{0}^{-1} v_{1}=A_{0}^{-1}\left(A_{0}^{-(k-1)} f\right)=A_{0}^{-k} f,
\end{aligned}
$$


which is (19) with

$$
A_{0}^{-k}=\underbrace{A_{0}^{-1} A_{0}^{-1} \cdots A_{0}^{-1}}_{k}
$$

Finally, since $R\left(A_{0}^{-k}\right)=X$ and $A_{0}^{-k}$ is bounded as a composition of bounded operators, it is concluded that the operator $A_{0}^{k}$ is correct.

For the important category of boundary value problems for $k=2$, we state the following corollary, which follows immediately from Theorem 3.

Corollary 1. The boundary value problem

$$
\begin{aligned}
A_{0}^{2} u & =A^{2} u=f, \\
D\left(A_{0}^{2}\right) & =\left\{u: u \in D\left(A^{2}\right), \mathrm{Y}(u)=0, \mathrm{Y}(A u)=\mathbf{0}\right\},
\end{aligned}
$$

is correct if and only if $\operatorname{det} Y(\mathbf{z}) \neq 0$ and the unique solution, for every $f \in X$, is given by

$$
\begin{aligned}
u= & A_{0}^{-2} f \\
= & \widehat{A}^{-2} f-\mathbf{z} \mathrm{Y}^{-1}(\mathbf{z}) \mathrm{Y}\left(\widehat{A}^{-2} f\right) \\
& -\left[\widehat{A}^{-1} \mathbf{z}-\mathbf{z} \mathrm{Y}^{-1}(\mathbf{z}) \mathrm{Y}\left(\widehat{A}^{-1} \mathbf{z}\right)\right] \mathrm{Y}^{-1}(\mathbf{z}) \mathrm{Y}\left(\widehat{A}^{-1} f\right)
\end{aligned}
$$

\subsection{Product of Two Operators}

Here, we are looking at yet another special boundary value problem, which is the generalization of Corollary 1. In particular, we consider the boundary value problem

$$
\begin{aligned}
A_{0} \tilde{A}_{0} u & =A^{2} u=f, \quad x \in(a, b), \\
D\left(A_{0} \tilde{A}_{0}\right) & =\left\{u: u \in D\left(A^{2}\right), \tilde{Y}(u)=\mathbf{0}, \mathrm{Y}(A u)=\mathbf{0}\right\},
\end{aligned}
$$

where $A, A_{0}$ are defined as in (1) and (9), respectively, and $\tilde{A}_{0}: X \rightarrow X$ is a restriction of $A$ defined by

$$
\begin{aligned}
\tilde{A}_{0} u & =A u, \quad x \in(a, b), \\
D\left(\tilde{A}_{0}\right) & =\{u: u \in D(A), \tilde{Y}(u)=\mathbf{0}\},
\end{aligned}
$$

wherein $\tilde{Y}=\operatorname{col}\left(\tilde{Y}_{1}, \ldots, \tilde{Y}_{n}\right)$ with $\tilde{Y}_{i} \in\left[X^{n-1}\right]^{*}$ being defined by

$$
\tilde{Y}_{i}(u)=\sum_{j=0}^{m} \sum_{k=0}^{n-1} \tilde{v}_{i k j} u^{(k)}\left(x_{j}\right)+\sum_{k=0}^{n-1} \int_{a}^{b} \tilde{h}_{i k}(t) u^{(k)}(t) d t, \quad i=1, \ldots, n,
$$

where $\tilde{h}_{i k}(x), i=1, \ldots, n, k=0, \ldots, n-1$, are continuous functions on $[a, b], \tilde{v}_{i k j}, i=$ $1, \ldots, n, k=0, \ldots, n-1, j=0, \ldots, m$, are constants.

Theorem 4. Let $A_{0}$ and $\tilde{A}_{0}$ be the linear operators defined in (22), $\mathbf{z}=\left(z_{1}, \ldots, z_{n}\right)$ a basis of $\operatorname{ker} A$, and $\widehat{A}^{-1}$ the inverse of the correct operator $\widehat{A}$ in (8). Then:

(i) The operator $A_{0} \tilde{A}_{0}$ is injective if and only if

$$
\operatorname{det} \mathrm{Y}(\mathbf{z}) \neq 0, \quad \operatorname{det} \tilde{Y}(\mathbf{z}) \neq 0 \text {. }
$$

(ii) Furthermore, under (24), the operator $A_{0} \tilde{A}_{0}$ is correct and the unique solution to the boundary value problem (22), for any $f \in X$, is given by

$$
\begin{aligned}
u= & \widehat{A}^{-2} f-\mathbf{z} \tilde{Y}^{-1}(\mathbf{z}) \tilde{Y}\left(\widehat{A}^{-2} f\right) \\
& -\left[\widehat{A}^{-1} \mathbf{z}-\mathbf{z} \tilde{Y}^{-1}(\mathbf{z}) \tilde{Y}\left(\widehat{A}^{-1} \mathbf{z}\right)\right] \mathrm{Y}^{-1}(\mathbf{z}) \mathrm{Y}\left(\widehat{A}^{-1} f\right)
\end{aligned}
$$


Proof. (i)-(ii) By setting $\tilde{A}_{0} u=v$, the problem (22) may be decomposed into the following two boundary value problems:

$$
\begin{array}{llll}
A v=f, & \mathrm{Y}(v)=\mathbf{0} & \text { or } & A_{0} v=f, \\
A u=v, & \tilde{Y}(u)=\mathbf{0} & \text { or } & \tilde{A}_{0} u=v .
\end{array}
$$

By Theorem 1, the boundary value problem (26) is correct if and only if $\operatorname{det} Y(\mathbf{z}) \neq 0$ and its unique solution is given by

$$
v=A_{0}^{-1} f=\widehat{A}^{-1} f-\mathbf{z} Y^{-1}(\mathbf{z}) Y\left(\widehat{A}^{-1} f\right) .
$$

Similarly, the boundary value problem (27) is correct if and only if $\operatorname{det} \tilde{Y}(\mathbf{z}) \neq 0$ and its solution is

$$
u=\tilde{A}_{0}^{-1} v=\widehat{A}^{-1} v-\mathbf{z} \tilde{Y}^{-1}(\mathbf{z}) \tilde{Y}\left(\widehat{A}^{-1} v\right) .
$$

Substitution of (28) into (29) yields

$$
\begin{aligned}
u= & \widehat{A}^{-1}\left[\widehat{A}^{-1} f-\mathbf{z} Y^{-1}(\mathbf{z}) \mathrm{Y}\left(\widehat{A}^{-1} f\right)\right] \\
& -\mathbf{z} \tilde{Y}^{-1}(\mathbf{z}) \tilde{Y}\left(\widehat{A}^{-1}\left[\widehat{A}^{-1} f-\mathbf{z} Y^{-1}(\mathbf{z}) \mathrm{Y}\left(\widehat{A}^{-1} f\right)\right]\right) \\
= & \widehat{A}^{-2} f-\widehat{A}^{-1} \mathbf{z} Y^{-1}(\mathbf{z}) \mathrm{Y}\left(\widehat{A}^{-1} f\right) \\
& -\mathbf{z} \tilde{Y}^{-1}(\mathbf{z}) \tilde{Y}\left(\widehat{A}^{-2} f\right)+\mathbf{z} \tilde{Y}^{-1}(\mathbf{z}) \tilde{Y}\left(\widehat{A}^{-1} \mathbf{z}\right) \mathrm{Y}^{-1}(\mathbf{z}) \mathrm{Y}\left(\widehat{A}^{-1} f\right) \\
= & \widehat{A}^{-2} f-\mathbf{z} \tilde{Y}^{-1}(\mathbf{z}) \tilde{Y}\left(\widehat{A}^{-2} f\right) \\
& -\left[\widehat{A}^{-1} \mathbf{z}-\mathbf{z} \tilde{Y}^{-1}(\mathbf{z}) \tilde{Y}\left(\widehat{A}^{-1} \mathbf{z}\right)\right] \mathrm{Y}^{-1}(\mathbf{z}) \mathrm{Y}\left(\widehat{A}^{-1} f\right),
\end{aligned}
$$

which is (25). Thus, the operator $A_{0} \tilde{A}_{0}$ is correct if and only if $\operatorname{det} Y(\mathbf{z}) \neq 0$ and $\operatorname{det} \tilde{Y}(\mathbf{z}) \neq 0$, and the unique solution of $A_{0} \tilde{A}_{0} u=f$ is given explicitly by (25).

\section{Examples}

To explain the implementation of the results presented in the previous section and to show the efficiency of the proposed solution routine, we solve two example problems.

Example 1. Consider the differential Equation [29]

$$
u^{\prime \prime}(x)+u(x)=0, \quad 0<x<\frac{\pi}{2}
$$

with the constraints

$$
\int_{0}^{\pi / 2} u(x) d x=1, \quad \int_{0}^{\pi / 2} u(x) \sin x d x=1 .
$$

Comparing with (12), it is natural to take

$$
\begin{aligned}
A u(x) & =u^{\prime \prime}(x)+u(x), \quad D(A)=C^{2}\left[0, \frac{\pi}{2}\right], \quad f(x)=0, \\
\mathrm{Y}_{1}(u(x)) & =\int_{0}^{\pi / 2} u(x) d x, \quad \mathrm{Y}_{2}(u(x))=\int_{0}^{\pi / 2} u(x) \sin x d x, \quad \beta_{1}=\beta_{2}=1,
\end{aligned}
$$

and $\widehat{A}$ and $A_{1}$ defined on

$$
\begin{aligned}
D(\widehat{A}) & =\left\{u: u \in D(A), u(0)=u^{\prime}(0)=0\right\}, \\
D\left(A_{1}\right) & =\{u: u \in D(A), \mathrm{Y}(u)=\mathbf{b}\},
\end{aligned}
$$

respectively. Observe that the only solution of $\widehat{A} u=0$ is $u \equiv 0, z_{1}=\cos x$ and $z_{2}=\sin x$ are two linearly independent solutions of $A u=0$, and the matrix 


$$
\mathrm{Y}(\mathbf{z})=\left(\begin{array}{ll}
\mathrm{Y}_{1}\left(z_{1}\right) & \mathrm{Y}_{1}\left(z_{2}\right) \\
\mathrm{Y}_{2}\left(z_{1}\right) & \mathrm{Y}_{2}\left(z_{2}\right)
\end{array}\right)
$$

is non-singular. Thus, by applying Theorem 2, we get the unique solution

$$
u(x)=\mathbf{z} Y^{-1}(\mathbf{z}) \mathbf{b}=\frac{2 \sin x+(\pi-4) \cos x}{\pi-2} .
$$

Example 2. Let the second order ordinary differential equation

$$
u^{\prime \prime}(x)-\frac{6}{x} u^{\prime}(x)+\frac{12}{x^{2}} u(x)=2 x^{4}, \quad x \in(1,2),
$$

subjected to non-local boundary conditions

$$
\begin{aligned}
8 u\left(\frac{3}{2}\right)-\frac{3}{4} u(2) & =\frac{177}{22} \int_{1}^{2} \frac{1}{t^{3}} u(t) d t \\
u^{\prime}\left(\frac{5}{4}\right)-\frac{12}{5} u\left(\frac{5}{4}\right) & =\frac{625}{768} \int_{1}^{2}\left(\frac{1}{t^{2}} u^{\prime}(t)-\frac{3}{t^{3}} u(t)\right) d t .
\end{aligned}
$$

Observe that this problem can be written as follows:

$$
\begin{aligned}
& {\left[\frac{d}{d x}-\frac{3}{x}\right]^{2} u(x)=2 x^{4}, \quad x \in(1,2)} \\
& 8 u\left(\frac{3}{2}\right)-\frac{3}{4} u(2)-\frac{177}{22} \int_{1}^{2} \frac{1}{t^{3}} u(t) d t=0 \\
& {\left.\left[\frac{d}{d x}-\frac{3}{x}\right] u(x)\right|_{x=\frac{5}{4}}-\frac{625}{768} \int_{1}^{2} \frac{1}{t^{2}}\left(\left[\frac{d}{d t}-\frac{3}{t}\right] u(t)\right) d t=0 .}
\end{aligned}
$$

Comparing now with (22), we take

$$
\begin{aligned}
A u(x) & =\left[\frac{d}{d x}-\frac{3}{x}\right] u(x), \quad D(A)=C^{1}[1,2] \\
f(x) & =2 x^{4} \\
\tilde{Y}(u) & =8 u\left(\frac{3}{2}\right)-\frac{3}{4} u(2)-\frac{177}{22} \int_{1}^{2} \frac{1}{t^{3}} u(t) d t, \\
Y(A u) & =\left.A u(x)\right|_{x=\frac{5}{4}}-\frac{625}{768} \int_{1}^{2} \frac{1}{t^{2}} A u(t) d t
\end{aligned}
$$

and we set

$$
\begin{aligned}
& \tilde{A}_{0} u=A u, \quad D\left(\tilde{A}_{0}\right)=\{u: u \in D(A), \tilde{Y}(u)=0\} \\
& A_{0} v=A v, \quad D\left(A_{0}\right)=\{v: v \in D(A), Y(v)=0\} .
\end{aligned}
$$

Further, let the correct operator $\widehat{A}$ defined by

$$
\widehat{A} u=A u=f, \quad D(\widehat{A})=\{u: u \in D(A), u(1)=0\},
$$

and its inverse given by

$$
\widehat{A}^{-1} f(x)=e^{\int_{1}^{x} \frac{3}{s} d s} \int_{1}^{x} e^{-\int_{1}^{t} \frac{3}{s} d s} f(t) d t=x^{3} \int_{1}^{x} \frac{1}{t^{3}} f(t) d t .
$$

Finally, note that $z(x)=x^{3}$ is a fundamental solution of the homogeneous equation $A u=0$ and that

$$
\mathrm{Y}(z) \neq 0, \quad \tilde{Y}(z) \neq 0 .
$$


Hence, from Theorem 4, it follows that the non-local boundary value problem (30), (31) has a unique solution, which after substituting into the formula (25), is

$$
u(x)=\frac{x^{3}\left(x^{3}-1\right)}{3} .
$$

\section{Discussion}

A method for constructing closed-form solutions to boundary value problems for ordinary differential equations with general multipoint and integral boundary conditions has been presented. Ready to use solution formulae in a symbolic form have been derived for some classes of boundary value problems. Specifically, we considered the following boundary value problems:

$$
\begin{aligned}
A_{0} u & =f, \quad D\left(A_{0}\right)=\{u: u \in D(A), \mathrm{Y}(u)=\mathbf{0}\}, \\
A_{1} u & =f, \quad D\left(A_{1}\right)=\{u: u \in D(A), \mathrm{Y}(u)=\mathbf{b}\}, \\
A_{0}^{k} u & =f, \quad D\left(A_{0}^{k}\right)=\left\{u: u \in D\left(A^{k}\right), \mathrm{Y}(u)=\mathbf{0}, \mathrm{Y}(A u)=\mathbf{0}, \ldots, \mathrm{Y}\left(A^{k-1} u\right)=\mathbf{0}\right\}, \\
A_{0} \tilde{A}_{0} u & =f, \quad D\left(A_{0} \tilde{A}_{0}\right)=\left\{u: u \in D\left(A^{2}\right), \tilde{Y}(u)=\mathbf{0}, \mathrm{Y}(A u)=\mathbf{0}\right\},
\end{aligned}
$$

where the operators $A_{0}, A_{1}, \tilde{A}_{0}$ are restrictions of the $n$-th order linear differential operator $A$ in (1) and $Y, \tilde{Y}$ are vectors of linear bounded functionals as in (4) and (23), respectively, describing general non-local boundary conditions.

The proposed methodology can be specialized to other categories of boundary value problems and extended to some classes of partial differential equations.

Author Contributions: Conceptualization, E.P., S.Z. and I.F.; validation, S.Z. and I.F.; formal analysis, E.P. All authors have read and agreed to the published version of the manuscript.

Funding: This research received no external funding.

Acknowledgments: The authors would like to thank the anonymous reviewers for their valuable suggestions and comments.

Conflicts of Interest: The authors declare no conflict of interest.

\section{References}

1. Busenberg, S.; Cooke, K.L. The effect of integral conditions in certain equations modelling epidemics and population growth. J. Math. Biol. 1980, 10, 13-32. [CrossRef] [PubMed]

2. Picone, M. Sui valori eccezionali di un parametro da cui dipende un'equazione dif- ferenziale lineare del secondo ordine. Annali della Scuola Normale Superiore di Pisa-Classe di Scienze 1910, 11, 1-141. (In Italian)

3. Sommerfeld, A. Ein Beitrag zur hydrodynamischen Erklärung der turbulenten Flüssigkeitsbewegung. Proc. Int. Math. Congr. 1909, 3, 116-124.

4. Tamarkin, J. Some general problems of the theory of ordinary linear differential equations and expansion of an arbitrary function in series of fundamental functions. Math. Z. 1928, 27, 1-54. [CrossRef]

5. Il'in, V.A.; Moiseev, E.I. An a priori estimate for the solution of a problem associated with a nonlocal boundary value problem of the first kind. Differ. Equ. 1988, 24, 519-526.

6. Dezin, A.A. On the general theory of boundary value problems. Math. USSR-Sb. 1976, 100, 171-180. [CrossRef]

7. Grubb, G. A characterization of the nonlocal boundary value problems associated with an elliptic operator. Annali della Scuola Normale Superiore di Pisa-Classe di Scienze 1968, 22, 425-513.

8. Vishik, M.I. On general boundary problems for elliptic differential equations. Am. Math. Soc. Transl. 1952, 1, 187-246.

9. Gorbachuk, M.L. Boundary Value Problems for Operator Differential Equations; Springer: Berlin, Germany, 1991. [CrossRef]

10. Krein, S.G. Linear Equations in Banach Spaces; Birkhäuser: Basel, Switzerland, 1982. [CrossRef]

11. Lions, J.L.; Magenes, E. Non-Homogeneous Boundary Value Problems and Applications; Springer: Berlin/Heidelberg, Germany, 1972; Volume 1. [CrossRef]

12. Skubachevskii, A.L. Nonclassical boundary-value problems. Int. J. Math. Sci. 2008 155, 199-334. [CrossRef]

13. Krall, A.M. The development of general differential and general differential-boundary systems. Rocky Mt. J. Math. 1975, 5, 493-542. [CrossRef]

14. Ma, R. A survey on nonlocal boundary value problems. Appl. Math. E-Notes 2007, 7, 257-279. 
15. Štikonas, A. A survey on stationary problems, Green's functions and spectrum of Sturm-Liouville problem with nonlocal boundary conditions. Nonlinear Anal.-Model. Control 2014, 19, 301-334. [CrossRef]

16. Whyburn, W.M. Differential equations with general boundary conditions. Bull. Am. Math. Soc. 1942, 48, 692-704. [CrossRef]

17. Denche, M.; Kourta, A. Boundary value problem for second-order differential operators with nonregular integral boundary conditions. Rocky Mt. J. Math. 2006, 36, 893-913. [CrossRef]

18. Gallardo, J.M. Second-Order differential operators with integral boundary conditions and generation of analytic semigroups. Rocky Mt. J. Math. 2000, 30, 1265-1291. [CrossRef]

19. Jankowski, T. Differential equations with integral boundary conditions. J. Comput. Appl. Math. 2002, 147, 1-8. [CrossRef]

20. Jones, W.R. Differential systems with integral boundary conditions. J. Differ. Equ. 1967, 3, 191-202. [CrossRef]

21. Kalenyuk, P.I.; Kuduk, G.; Kohut, I.V.; Nytrebych, Z.M. Problem with integral conditions for differential-operator equation. J. Math. Sci. 2015, 208, 267-276. [CrossRef]

22. Liu, L.; Hao, X. ; Wu Y. Positive solutions for singular second order differential equations with integral boundary conditions. Math. Comput. Model. 2013, 57, 836-847. [CrossRef]

23. Zhang, L.; Xuan, Z. Multiple positive solutions for a second-order boundary value problem with integral boundary conditions. Bound. Value Probl. 2016, 60. [CrossRef]

24. Zhang, X.; Ge, W. Positive solutions for a class of boundary-value problems with integral boundary conditions. Comput. Math. Appl. 2009, 58, 203-215. [CrossRef]

25. Adomian, G. Integral Boundary Conditions. In Solving Frontier Problems of Physics: The Decomposition Method. Fundamental Theories of Physics (An International Book Series on the Fundamental Theories of Physics: Their Clarification, Development and Application); Springer: Dordrecht, The Netherlands, 1994; Volume 60, pp. 196-210. [CrossRef]

26. Chen, S.; Ni, W.; Wang, C. Positive solution of fourth order ordinary differential equation with four-point boundary conditions. Appl. Math. Lett. 2006, 19, 161-168. [CrossRef]

27. Dovletov, D.M. On a nonlocal boundary value problem of the second kind for the Sturm- Liouville operator in the differential and difference statements. e-J. Anal. Appl. Math. 2018, 1, 37-55. [CrossRef]

28. Krall, A.M. Differential operators and their adjoints under integral and multiple point boundary conditions. J. Differ. Equ. 1968, 4, 327-336. [CrossRef]

29. Ojika, T.; Welsh, W. A numerical method for the solution of multi-point problems for ordinary differential equations with integral constraints. J. Math. Anal. Appl. 1979, 72, 500-511. [CrossRef]

30. Parasidis, I.N.; Providas, E. Exact solutions to problems with perturbed differential and boundary operators. In Analysis and Operator Theory; Springer Optimization and Its Applications; Rassias, T., Zagrebnov, V., Eds.; Springer: Cham, Switzerland, 2019; Volume 146. pp. 301-317. [CrossRef]

31. Sadybekov, M.A.; Imanbaev, N.S. A regular differential operator with perturbed boundary condition. Math. Notes 2017, 101, 878-887. [CrossRef]

32. Aida-Zade, K.R.; Abdullaev, V.M. On the solution of boundary value problems with nonseparated multipoint and integral conditions. Differ. Equ. 2013, 49, 1114-1125. [CrossRef]

33. Biyarov, B.N. Normal extensions of linear operators. Eurasian Math. J. 2016, 7 17-32.

34. Kokebaev, B.K.; Otelbaev, M.; Shynibekov, A.N. About Restrictions and Extensions of operators. DAN SSSR 1983, 271, 1307-1310. (In Russian)

35. Oinarov, R.O.; Parasidis, I.N. Correct extensions of operators with finite defect in Banach spaces. Izv. Akad. Kaz. SSR 1988 5, $42-46$. (In Russian)

36. Parasidis, I.N.; Tsekrekos, P. Some quadratic correct extensions of minimal operators in Banach spaces. Oper. Matrices 2010, 4, 225-243. [CrossRef]

37. Parasidis, I.N.; Providas, E.; Zaoutsos, S. On the Solution of Boundary Value Problems for Ordinary Differential Equations of Order $\mathrm{n}$ and 2n with General Boundary Conditions. In Computational Mathematics and Variational Analysis; Springer Optimization and Its Applications; Daras, N., Rassias, T., Eds.; Springer: Cham, Switzerland, 2020; Volume 159, pp. 299-314. [CrossRef] 